\title{
REFINING THE MODELLING OF VEHICLE-TRACK INTERACTION
}

\author{
Ingo Kaiser \\ Institute of Robotics and Mechatronics, German Aerospace Center (DLR) Oberpfaffenhofen \\ Muenchner Strasse 20 \\ D-82234 Wessling, Germany \\ Ingo.Kaiser@dlr.de
}

\begin{abstract}
A refined model of a railway vehicle running on a straight track is developed. In this model the wheelsets and the rails are modelled as flexible bodies and a detailed model of the wheel-rail contact is used. The simulation results obtained with this model show a distinct impact of the structural deformations on the stress distributions in the contact. This refined model can serve as a base for investigations of rolling noise and wear.
\end{abstract}

\section{Introduction}

Today, modelling of railway vehicles as multi-body systems (MBS) covers a wide range of problems, e.g. running stability or curving behaviour. The MBS modelling describes the real system by a combination of rigid bodies, whereas their motions of the bodies are determined by joints and constraints, and force elements acting between the bodies. However, there are at least two phenomena related to the vehicle-track interaction, for which the MBS modelling seems to be insufficient.

The first phenomenon is the rolling noise, resulting from structural vibrations of wheels and rails which are excited by disturbances and irregularities of the running surfaces. It is obvious that a description of this phenomenon requires the consideration of the wheels' and the rails' structural dynamics, i.e. these components have to be modelled as flexible bodies.

The second phenomenon is wear. It is obvious that the wear is related to the noise: Irregularities which are responsible for high-frequent structural vibrations like e.g. corrugation are caused by wear, and it can also be assumed that these vibrations have an impact on the wear. Therefore, the description of wheelsets and rails as flexible bodies is also desirable in this context. But the modelling of the wear also requires a more detailed model of the wheel-rail contact: Many commercially available simulation programs assume a Hertzian contact between wheel and rail, i.e. an elliptically shape contact area, although contact areas of real wheel-rail profiles can deviate from an ellipse, even for newly manufactured profiles. The assumption of an elliptical contact area may be sufficient, if only the resulting forces are of interest. However, for describing wear a more detailed knowledge of the actual contact area is necessary, because abrasive wear can only occur, where the wheel and the rail are actually in contact. From a more accurate description of the stress distribution in the contact, stresses acting inside the wheel rim and the rail head can be derived. Such stresses are relevant for phenomena like rolling contact fatigue $(\mathrm{RCF})$.

In this work, a refined model of a railway vehicle is developed, which contains the enhancements of flexible wheelsets and rails and a more detailed contact model. Here, acoustics and wear are not yet modelled, but the interaction between the flexibility of wheelsets and rails and the will be investigated.

\section{Description of the model}

The model represents a railway passenger coach running on a straight track. An overview of the entire vehicletrack model and the modifications compared to the standard modelling is given in Fig. 1. Since the wheelsets, the rails, and the wheel-rail contact are the key components of the vehicle-track interaction, these components are enhanced compared to the commercially available "standard modelling".

The motions of the flexible wheelset are described by superposing the motions of the undeformed body, the socalled rigid body motions, and the deformations, which are expressed by a modal synthesis. Eigenmodes of the wheelset, which are obtained from a three-dimensional (3D) Finite Element (FE) model, are used as shape functions for the modal synthesis. The wheelset is a rotational symmetric structure, which allows the application of a semi-analytical solution, being a product of trigonometric functions in the circumferential direction and local bilinear functions in the longitudinal plain, as shown in Fig. 2. The mathematical description is given in [1]. The symmetry of the wheelset with respect to the middle cross plain allows a further reduction without loss of accuracy. - The main difficulty is the description of the wheel-rail forces acting on the wheelset, because the wheel-rail contact is permanently moving around the wheelset. Therefore, the motions of the wheelset are 
described in an intermediate frame, which performs all the rigid body motions except the overturning motion. The exploitation of some characteristics of the wheelset's rotationally symmetric shape provides a simple, but mathematically exact description of the rotating flexible wheelsets with full consideration of the gyroscopic effects. A detailed description and derivation of this method can be found in [2].

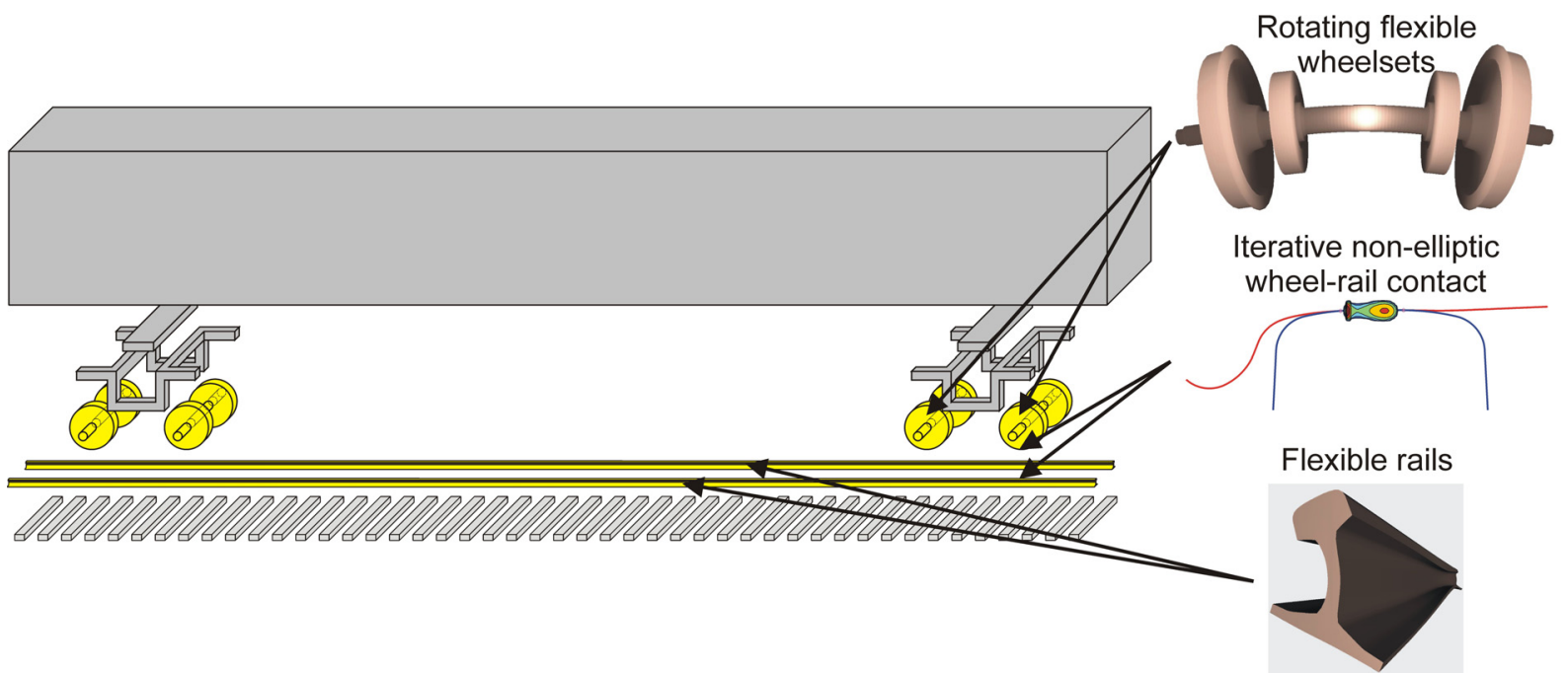

Figure 1: Overview of the refined vehicle-track system. Yellow bodies are modelled as flexible bodies
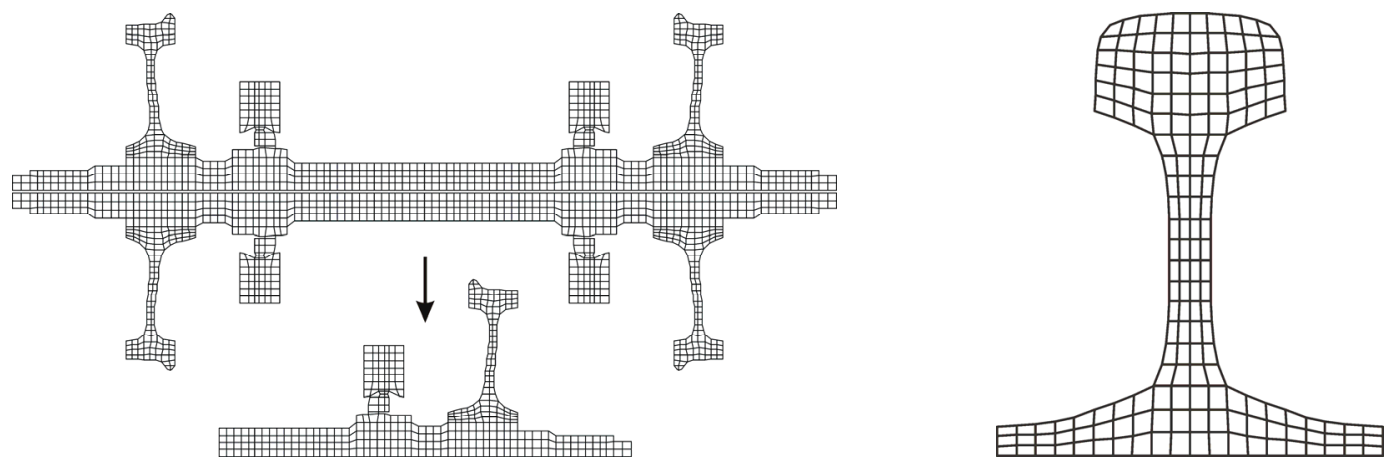

Figure 2: Finite Element discretisation of the half wheelset (490 nodes) and the rail's cross section (216 nodes)

The track model is based on the track model developed by Ripke [3], but is enhanced in some aspects. It consists of two flexible rails, which are supported by discrete sleepers. The deformations of the rails are described by a modal synthesis. The shape functions are selected eigenmodes obtained from a 3D FE model, which takes also deformations of the cross section into account. Similar to FE model of the wheelset, a semi-analytical solution is used so that only the cross section has to be discretised by local bilinear functions, see Fig. 2, while trigonometric functions for the longitudinal direction are used, see [1] for the mathematical details. The geometry of the rail represents the profile UIC60. The inclination of 1/40 is taken into account in the model. The sleepers are modelled as rigid bodies. They are connected to the rails and to the ground by distributed linear springs and dampers, representing the pads and the underground, respectively. To approximate an infinite track, the boundary conditions at the rails' ends are set equal, so that the track forms a "ring with neglected curvature". For a suitable approximation of the infinite track, a length of 128 sleeper bays or $76.8 \mathrm{~m}$ is used. For the entire track model, a modal decomposition is performed. The most influential modes are transferred into the vehicletrack system. Since the longitudinal distribution of the deformations at the rail head are expressed by trigonometric functions, i.e. continuous analytical functions, the description of the moving loads along the track is relatively simple.

From the point of view of MBS modelling, the wheel-rail contact is a force element, i.e. its inputs are the relative kinematics between the wheel rim and the rail head, its outputs are the resulting forces acting between the wheel and the rail. Here, the profiles S1002 and UIC60 are used for the wheel and the rail, respectively. Since nonelliptic contact areas can occur for this profile combination, the contact model should to be able to describe such areas. There are several possibilities to deal with non-elliptic contact areas. One possibility is to estimate the contact area based on the interpenetration of the undeformed surfaces of wheel and rail, see e.g. the survey in [4]. Another possibility is to solve the equations of a model describing the contact mechanics. Such a model can be 
derived on the base of the Finite Element Method (FEM) or of the Boundary Element Method (BEM). Here, the BEM is chosen for the model, which is mainly based on the fundamentals given by Kalker in [5]. Since the contact area is assumed to be small compared to the main dimensions of the wheel and the rail, both bodies are considered as halfspaces. The normal pressure $p(x, y)$ and the tangential stresses $\tau_{1}(x, y)$ and $\tau_{2}(x, y)$ are discretised by using local bilinear functions on an equidistant grid, as shown in Fig. 3. Inserting the discretised distributions of the stresses into the Boussinesq-Cerrutti equations, see [5], leads to a system of linear equations, which gives a relation between the stresses and the deformations at the grid points. The results presented in the following were obtained for a discretisation using a spacing of $\Delta a=0.75 \mathrm{~mm}$. The system of equations is solved iteratively. Therefore, the wheel-rail contact model contains an actual calculation of the stresses.
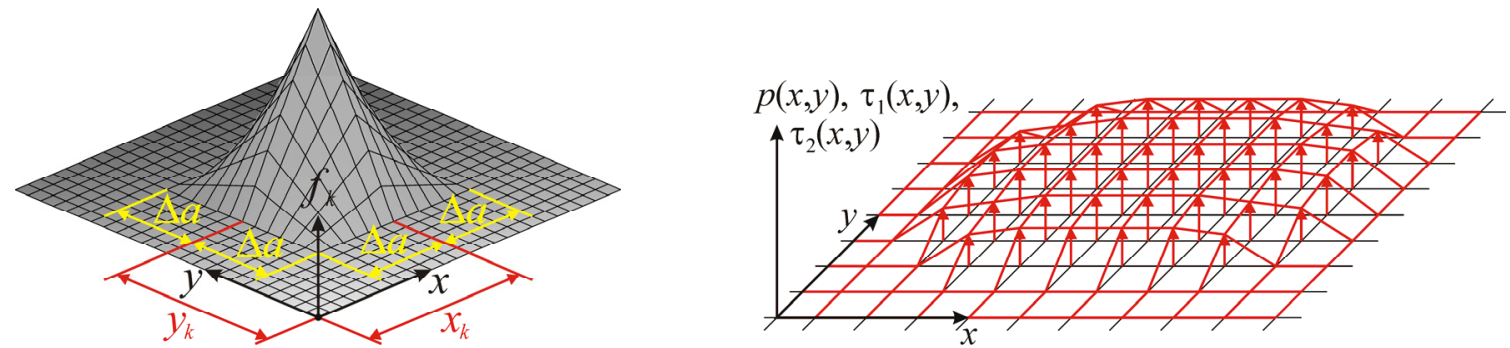

Figure 3: Local bilinear function (left) and discretised stress distribution (right)

The complete model described above is implemented in a program written in FORTRAN. The modelling methods for the rotating flexible wheelsets and for the flexible track, which have been developed and tested with this program, have been successfully transferred into a developer version of the commercially available MBS simulation software SIMPACK.

\section{Results}

To study the influence of the structural deformations on the contact, two variants of the vehicle-track model are compared. The detailed model, as described before, takes the flexibility of the wheelsets and the rails into account. This model will be referred as "flexible model". For the comparison, a second model is used, where the wheelsets are described as rigid bodies. Furthermore, in this model each wheelset is supported by a simple track element consisting of a rigid body linked to the environment by springs and dampers, see Fig. 4. This variant will be called "rigid model".

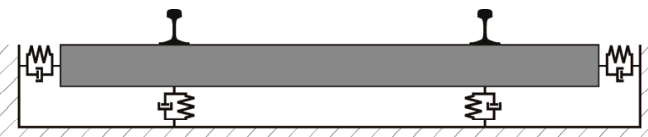

Figure 4: Simple track model ("rigid rails")

Two scenarios are studied, the undisturbed centred running and the hunting motion. In both cases, no track disturbances are taken into account. All results presented in the following are obtained for the front wheelset of the vehicle and its right wheel-rail contact. As mentioned above, the profiles S1002 and UIC60 are used. The friction coefficient is set to $\mu=0.3$.

In Fig. 5 the results for the case of undisturbed centred running at a driving speed of $\mathrm{v}_{0}=200 \mathrm{~km} / \mathrm{h}$ are displayed. The deformation of the wheelset and the rails has only a small impact on the contact geometry. Differences are hardly visible. However, even this small change has a distinct impact on the contact. While the distribution of the normal pressure shows two maxima for the rigid model, the left maximum vanishes nearly completely for the flexible model. The distribution of the tangential stresses clearly shows the influence of the spin resulting from the inclination of the contact relative to the rotation axis of the wheelset. The sliding zone at the trailing edge of the contact patch is visible. The single points indicating sliding at the leading edge result from discretisation errors. At the sliding zone, frictional power occurs, which is relevant for wear. The impact of the structural deformation on the frictional power density is remarkable: The left maximum occurring for the rigid model is distinctly reduced. The reason is that the normal pressure at the left maximum is drastically reduced for the flexible model, which causes a corresponding reduction of the friction stresses.

The impact of the deformations on the contact can be explained: The vertical forces acting at the bearings $\left(\mathrm{F}_{\mathrm{B}}\right)$ and at the wheel-rail contact $\left(\mathrm{F}_{\mathrm{WR}}\right)$ cause a deformation as depicted qualitatively in Fig. 6 . A bending of the axle 
and, thereby, an inclination of the wheels occur as indicated by the blue arrow. The resulting inclination of the wheel rim causes the change of the normal pressure distribution.

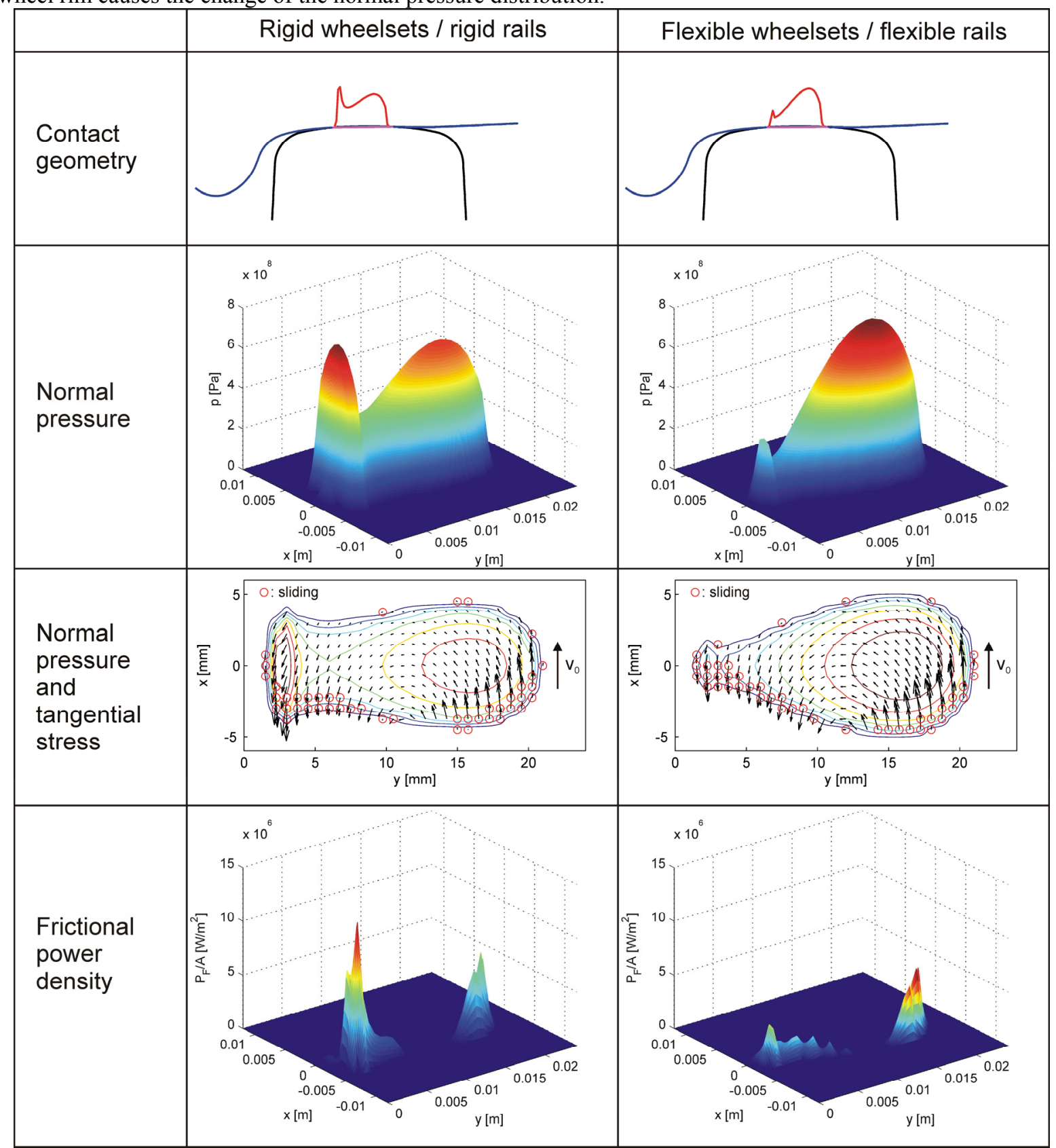

Figure 5: Wheel-rail contact for the rigid and the flexible model for centred running at $v_{0}=200 \mathrm{~km} / \mathrm{h}$

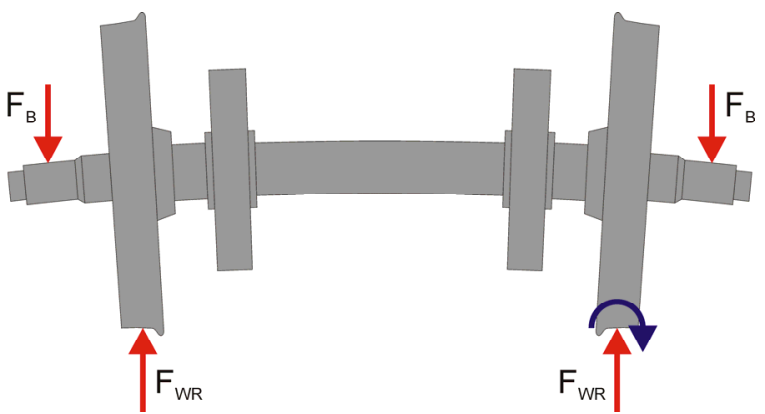

Figure 6: Deformation of the wheelset due to the loading by vertical bearing forces and wheel-rail forces

Furthermore, the scenario of permanent or "unstable" hunting is studied, i.e. the driving speed is higher than the critical speed of the vehicle. Although this case should be avoided in regular operation, it gives some insight into 
the system's behaviour. As a result, the phase portraits for the motion of the first wheelset's centre are shown in Fig. 7 for the rigid and for the flexible model for different values of the driving speed $\mathrm{v}_{0}$.
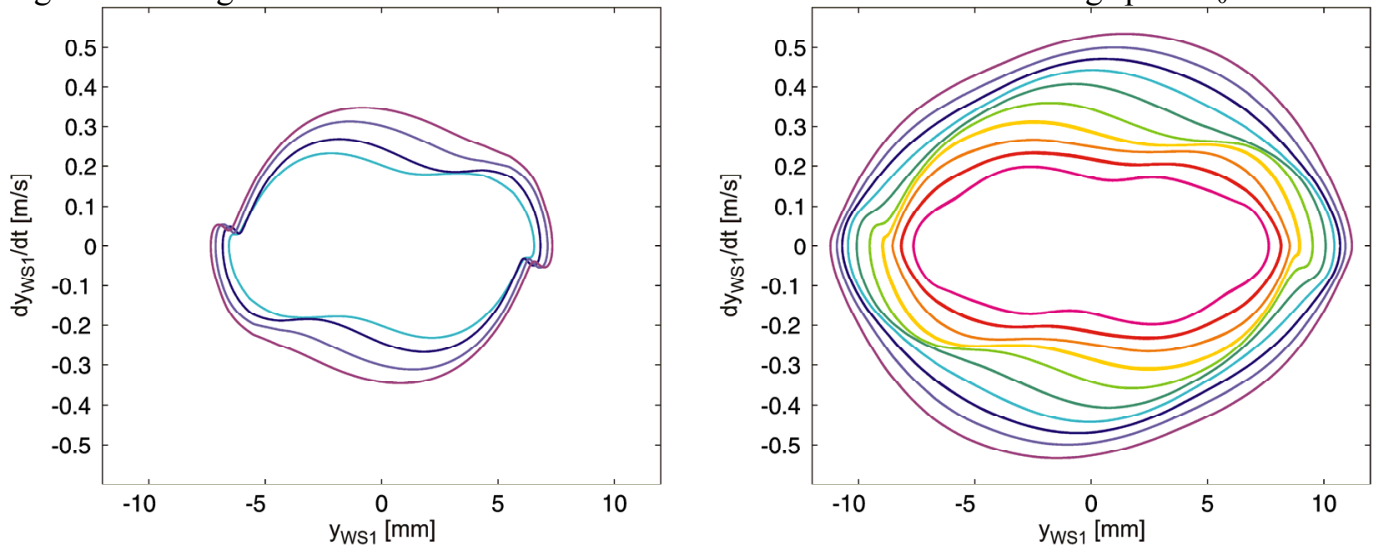

Figure 7: Phase portrait of the lateral motion of the wheelset's centre for rigid wheelsets and rails (left) and for flexible wheelsets and rails (right)

The comparison of the diagrams shows three main differences:

- The amplitudes for the flexible model are larger than for the rigid model, because due to the deformations the centre of the wheelset moves farther aside.

- The curves for the rigid model show distinct kinks at $\mathrm{y}_{\mathrm{ws} 1}= \pm 7 \mathrm{~mm}$, while the curves for the flexible model are smoother. These kinks occur, when the wheel's flange hits the rail's corner. If the structural dynamics of the wheelsets and the rails are taken into account, these bodies act as springs which are softer than the comparatively stiff wheel-rail contact. Thereby, the impact of the wheel flange is cushioned, and the kinks are smoothed.

- The curves for $\mathrm{v}_{0}=330 \mathrm{~km} / \mathrm{h}$ up to $\mathrm{v}_{0}=380 \mathrm{~km} / \mathrm{h}$ are missing in the left diagram, because the rigid model shows no permanent hunting for these driving speeds. In other words, the critical speed, above which permanent hunting occurs, is distinctly lower for the flexible model.

These differences show that the structural deformations of the wheelsets and the rails have in fact a distinct impact on the running behaviour, as it was already shown in an earlier work [1].

To study the influence on the contact, the position of the profiles and the pressure distribution are displayed in Fig. 8 for different values of the lateral displacement $y_{w s}$.

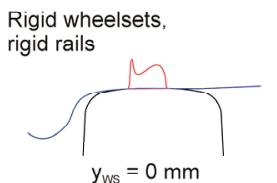

$\mathrm{y}_{\mathrm{ws}}=0 \mathrm{~mm}$

Flexible wheelsets. flexible rails

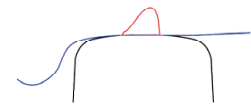

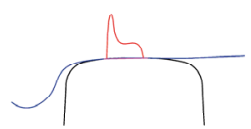

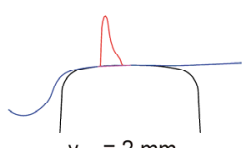

$\mathrm{y}_{\mathrm{wS}}=2 \mathrm{~mm}$

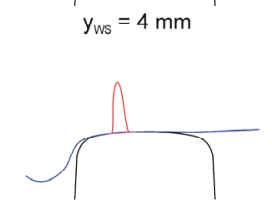

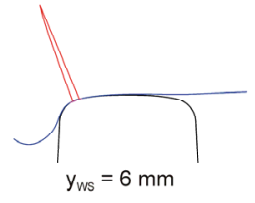
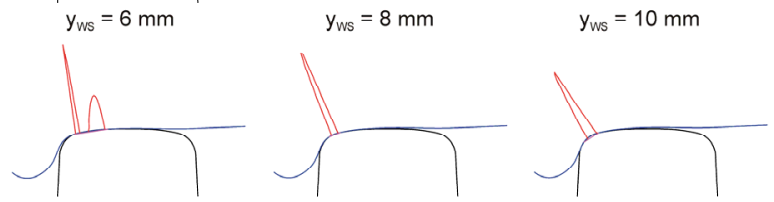

Figure 8: Contact geometry and distribution of the normal pressure depending on the wheelset's lateral motion

The impact of the structural deformations is clearly visible: The distributions of the normal pressure show distinct differences for the rigid and the flexible model. It can also be seen, that the differences of the contact geometry and the position of the contact area for the shifts of $\mathrm{y}_{\mathrm{WS} 1}=8 \mathrm{~mm}$ and $\mathrm{y}_{\mathrm{WS} 1}=10 \mathrm{~mm}$ are small. If the contact geometry is nearly unchanged, the shift is mainly caused by structural deformations. This strengthens the thesis mentioned above, that the structural flexibility cushions the impact in the case of flange contact.

\section{Conclusion}

The comparison of the results shows that the structural deformations of the wheelsets and the rails have in fact a distinct impact on the wheel-rail contact. Already for the "unspectacular" case of undisturbed centred running of the wheelset, a distinct change of the contact area and the stress distribution occurs, if the deformations of the 
wheelset and the rails are taken into account. This change also affects the distribution of the frictional power in the contact area which is relevant for wear.

In the scenario of permanent hunting, the running behaviour of the vehicle is distinctly influenced by the structural dynamics: The critical speed of the vehicle is about $50 \mathrm{~km} / \mathrm{h}$ lower for the flexible model. Furthermore, the structural deformations have an impact on the motion itself, because the flexibility of the wheelsets and the rails cushions the impact, when the wheel flange hits the rail head. Also in this case, the structural deformations cause distinct changes of the pressure distribution.

The impact of the structural deformations on the contact underlines that only a consistent refinement of the modelling is sensible. A more accurate calculation requires a more detailed calculation method as well as a more accurate input, in other words: A refined model of the wheel-rail contact is only useful, if it gains its input from refined models of the wheelset and the rail, while combining a detailed contact model with a rigid body model of wheelset and track doesn't seem to be worth the increased computational effort required by the contact model.

The vehicle-track model presented in this work contains the structural dynamics of the wheelsets and the rails as well as a very detailed model of the wheel-rail contact. This model may serve as a base for further investigations of acoustics and wear, where the structural vibrations of wheelsets and rails and the stresses acting in the contact play an essential role. The three refined components shall be made available in commercial simulation programs like the MBS simulation software SIMPACK. After being developed and tested in a prototype program, the descriptions of the rotating flexible wheelsets and of the flexible track have already been implemented into a developer version of this tool.

\section{References}

[1] I. Kaiser and K. Popp: Interaction of Elastic Wheelsets and Elastic Rails: Modeling and Simulation. Vehicle System Dynamics, Vol. 44, Supplement 2006, pp. 932-939.

[2] I. Kaiser, A. Heckmann and F. van der Linden: On an ALE-approach for rotating elastic structures. Proceedings of the MULTIBODY DYNAMICS 2007, ECCOMAS Thematic Conference, edited by C.L. Bottasso, P. Masarati, L. Trainelli. Milano, Italy, 25-28 June 2007.

[3] B. Ripke: Hochfrequente Gleismodellierung und Simulation der Fahrzeug-Gleis-Dynamik unter Verwendung einer nichtlinearen Kontaktmechanik. Fortschritt-Berichte VDI, Reihe 12, Nr. 249. VDI-Verlag, Düsseldorf 1995.

[4] J. Piotrowski and H. Chollet: Wheel-rail contact models for vehicle system dynamics including multi-point contact. Vehicle System Dynamics, Vol. 43, No. 6-7, June-July 2005, pp. 455-483

[5] J. J. Kalker: Three-dimensional elastic bodies in rolling contact. Kluwer Academic Publishers, Dordrecht 1990. 